\title{
La longue marche du Kenya vers la scolarisation universelle
}

\section{Hélène Charton}

\section{CpenEdition \\ Journals}

Édition électronique

URL : http://journals.openedition.org/ries/528

DOI : $10.4000 /$ ries.528

ISSN : 2261-4265

Éditeur

Centre international d'études pédagogiques

\section{Édition imprimée}

Date de publication : 1 avril 2009

Pagination : 139-144

ISSN : 1254-4590

\section{Référence électronique}

Hélène Charton, "La longue marche du Kenya vers la scolarisation universelle », Revue internationale d'éducation de Sèvres [En ligne], 50 | avril 2009, mis en ligne le 01 avril 2012, consulté le 01 mai 2019. URL : http://journals.openedition.org/ries/528 ; DOI : 10.4000/ries.528 


\section{Repères}

\section{LA LONGUE MARCHE DU KENYA VERS LA SCOLARISATION UNIVERSELLE}

Le Kenya contemporain se caractérise par l'importance du fait scolaire à la fois d'un point de vue social et politique. Que ce soit en ville ou dans les campagnes, le paysage est dominé par une multitude d'écoles; l'éducation est au cœur des discours politiques, particulièrement au moment des échéances électorales. Depuis son indépendance en 1963, le développement rapide de l'éducation au Kenya a été le fruit d'une volonté politique affirmée, relayée par un investissement majeur des communautés locales. Au cours des cinq dernières années, le pays s'est lancé, grâce à l'appui de la communauté internationale, dans un programme ambitieux de scolarisation universelle avec la rémission des frais de scolarité dans l'enseignement primaire en 2003, puis dans le secondaire en 2008. Ce renouveau scolaire, qui fait suite à la décennie de crise des années 1990, a généré d'importantes recompositions du système éducatif. Celles-ci ont modifié le rôle et la place de l'école dans la société.

\section{Les dynamiques historiques de la scolarisation au Kenya}

L'importance du fait scolaire au Kenya est en partie imputable à l'histoire particulière de cette ancienne colonie britannique. L'administration coloniale, sous l'influence d'un colonat blanc très puissant, avait mené une politique de développement séparé notamment dans le domaine de l'éducation. Chaque population de la colonie (Africains, Indiens et Européens) recevait une éducation correspondant à sa place dans l'édifice colonial. La scolarisation des Africains était confiée aux missionnaires qui s'intéressaient davantage à l'évangélisation des populations qu'à leur éducation. Ce parti pris avait rapidement suscité des conflits d'intérêts avec l'administration coloniale qui subventionnait ces écoles, mais aussi avec les populations africaines insatisfaites.

Certaines assemblées locales (Local Native Councils - LNC), créées en 1924 pour donner un canal légal d'expression aux revendications africaines et promouvoir le développement local, ont ainsi décidé de lever une taxe spéciale pour créer leurs propres écoles. Dans la province centrale du Kenya, plusieurs communautés kikuyu ont formé des églises et des écoles indépendantes en rupture avec les églises chrétiennes. À partir de 1945, la question scolaire devient une revendication politique majeure : la Kenya African Union (qui est la première organisation politique africaine d'envergure nationale) réclame ainsi la scolarisation gratuite et obligatoire. Cette pression sociale et politique semble porter ses fruits puisque la scolarisation triple entre 1949 et 1959. 
Les efforts déployés par les communautés en faveur de l'éducation se poursuivent après l'indépendance du pays, en 1963. L'école devient alors officiellement le levier du développement économique et identitaire de la nouvelle nation indépendante.

À l'instar de nombreux pays africains, l'indépendance est marquée par une véritable fièvre scolaire. Les écoles primaires de la jeune république du Kenya accueillent plus d'un million d'élèves en janvier 1964, soit deux fois plus qu'en 1957 (Bogonko, 1992). Répondant au mot d'ordre lancé par le président Jomo Kenyatta "Harambee, Harambee!» qui signifie en swahili «Tirons tous ensemble pour aller de l'avant ", les communautés se mobilisent pour construire des écoles. En 1978, le Kenya compte quatre millions d'élèves dans le primaire soit quatre fois plus qu'en 1963 et treize fois plus dans le secondaire (Eshiwani, 1993). Les familles contribuent activement à cette expansion du système scolaire à travers les frais de scolarité qui représentent, en 1970, un tiers du budget des écoles et les Harambee Funds qui servent à construire de nouvelles écoles et à payer les enseignants. En 1973, 60 \% des écoles secondaires sont des écoles Harambee ; lorsqu'elles ont atteint un niveau satisfaisant, ces écoles sont intégrées dans le système national. Entre 1963 et 1974, la part du budget de l'État consacrée à l'éducation passe de $11,9 \%$ à 24,7 \% (Abreu, 1982). C’est précisément l'État qui impulse cette dynamique collective originale en encourageant et en récompensant les initiatives des communautés. En 1973, à l'occasion des dix années d'indépendance, le président Kenyatta décrète la scolarisation gratuite pour les quatre premiers niveaux du primaire. Cette mesure, qui vient récompenser les efforts des populations, renvoie à un idéal social et politique très fort. La scolarisation universelle figurait dans le programme du parti KANU ${ }^{1}$ dès 1963. L'une des premières mesures adoptées par Daniel Arap Moi, devenu président en 1978, est l'extension de la gratuité de l'école à toutes les classes du primaire. Il légitime ainsi son action en l'inscrivant dans une certaine continuité historique. Ces décisions politiques entraînent un nouvel afflux d'élèves dans le primaire, dont le nombre augmente de $49 \%$ en janvier 1974. Ce recrutement exceptionnel décroît cependant rapidement car la gratuité de l'école n'est qu'apparente. Cette forte affluence nécessite des constructions de classes supplémentaires qui entraînent de nouvelles contributions comme les Activity fees, les School building funds, ou encore les Watchmen fees (Abreu, 1982)... Aucune loi ne régit ces participations. Aussi, d'une école à l'autre, les situations sont extrêmement variables, parfois, les frais de scolarité demandés sont plus élevés qu'avant la gratuité... Ces mesures génèrent également une forte demande au niveau du secondaire que les écoles du pays ne sont pas en mesure d'absorber. Par conséquent, le passage du premier au second cycle devient plus sélectif, renforçant ainsi le caractère élitiste du système scolaire. Or, en dépit de la contribution active de la population kenyane, la croissance de la population scolaire pèse lourdement sur le budget de l'État, à un moment où la conjoncture économique se retourne.

1. Kenya African National Union, parti politique dirigé par Jomo Kenyatta, vainqueur aux élections de 1963 et qui conserve le pouvoir jusqu'en 2002. 


\section{Crise et recomposition du système éducatif}

Les plans d'ajustement structurels adoptés pour faire face à la crise touchent directement le secteur éducatif. L'État, sommé de réduire ses dépenses, concentre ses efforts sur les besoins les plus urgents, en l'occurrence le niveau primaire. La réforme 8-4-4, adoptée en 1985, porte la durée du cycle primaire à huit années (contre sept auparavant). Les cycles secondaires et universitaires sont désormais de quatre années chacun au lieu de six et trois ans. Afin de rendre les enseignements plus adaptés aux besoins du pays, les disciplines techniques et agricoles sont réintroduites dans les programmes. Les communautés participent au financement de la réforme en contribuant à la construction de nouvelles classes et aux nouveaux équipements. En 1989, la scolarisation primaire universelle est pratiquement atteinte avec un taux brut de scolarisation de $105 \%$ (30\% dans le secondaire).

Les années 1990 sont cependant marquées par une forte déscolarisation : en 1998, les taux bruts de scolarisation tombent à $89 \%$ dans le primaire, et $23 \%$ dans le secondaire. Les populations appauvries par la crise économique ne peuvent plus assumer le coût de l'éducation qui ne cesse d'augmenter : entre 1994 en 1997, la charge revenant aux familles pour la scolarisation d'un élève dans le primaire est passée de 843 à $1200 \mathrm{Ksh}^{2}$. Or la part du budget de l'État consacrée à l'éducation continue de grimper, passant de $18,5 \%$ à $26,4 \%$ entre 1989 et 1999 (dont $90 \%$ vont aux traitements des enseignants). Ce système très coûteux n'est pas performant pour autant. En 1999, les taux de complétion

2. Ksh : shilling kenyan. du primaire sont de $40 \%$ mais ils sont seulement $40 \%$ à accéder au secondaire, contre $49 \%$ en 1992 (Koech, 2000). Ces résultats médiocres sont généralement imputés au système 8-4-4. Les programmes sont en effet très lourds, avec 13 matières dans le primaire et 32 dans le secondaire. La survalorisation de l'examen de fin d'études primaires, le KCPE (Kenya Certificate of Primary Education) qui détermine l'accès à l'éducation secondaire ${ }^{3}$ a des effets négatifs sur les derniers niveaux de primaire qui se transforment en classes de bachotage.

Mais cette crise est également porteuse de recompositions dont l'une des caractéristiques majeures est l'essor de l'enseignement privé. Alors qu'une part de la population se trouve pour la première fois exclue du système national d'enseignement, les écoles privées permettent aux plus riches de garantir à leurs enfants une éducation de qualité. En 1990, le Kenya comptait 290 écoles primaires privées, principalement situées en milieu urbain. Aujourd'hui, leur nombre a décuplé et on les trouve partout. Or, avec $1 \%$ seulement des élèves, les candidats de ces écoles ont pris les dix meilleures places et la moitié des cinquante premières au KCPE de 1996 ; cinq ans plus tard, en 2001, parmi les 30 écoles ayant obtenu les meilleurs scores au KCPE, 27 étaient privées, la première école publique arrivant seulement en quatrième position. Le succès de ces écoles est révélateur de la faillite d'un système public d'enseignement à bout de souffle. Officiellement, le gouvernement s'émeut de la situation et promet de garantir sa

3. Les meilleurs élèves intègrent les écoles secondaires nationales mieux équipées et plus performantes, tandis que les autres sont envoyés dans les écoles de district moins bien pourvues. 
politique égalitaire d'éducation avec des quotas limitant l'accès des élèves du privé aux lycées nationaux les plus performants. Mais les appels répétés aux investissements privés dans le domaine scolaire et les promesses de mesures fiscales avantageuses pour ces entrepreneurs soulignent combien l'enseignement privé fait désormais partie intégrante des stratégies de développement de l'éducation. De telles évolutions comportent le risque de voir émerger un système d'éducation à deux vitesses qui renforcerait les inégalités sociales et fragiliserait l'unité nationale qui reposait en partie sur ce consensus scolaire.

Les évolutions récentes de l'enseignement supérieur permettent d'apprécier les enjeux de ces processus de privatisation de l'éducation. L'introduction, en 1991, du principe du cost sharing with beneficiaries dans les lycées et les universités, a radicalement modifié le statut des étudiants qui ont dû, pour la première fois, acquitter des frais de scolarité. Par ailleurs, la création de la Commission for Higher Education (CHE) chargée d'habiliter les universités privées a encouragé leur développement dans tout le pays. Le Kenya compte aujourd'hui 7 universités publiques et 21 universités privées (dont 8 sont totalement autonomes et délivrent leurs propres diplômes); elles accueillent $17 \%$ des 118239 étudiantskenyans (Unesco, 2008). Le succès sans précédent des universités privées s'explique en partie par la saturation de leurs homologues publiques: moins de la moitié des lauréats du certificat d'études secondaires (le KCSE: Kenya Certificate of Secondary Education) sont admis dans les universités publiques ${ }^{4}$.

4. Le système universitaire est résidentiel et le nombre de places de chaque université dépend de son nombre de lits.
Pour pallier ces difficultés et générer de nouveaux revenus, les universités publiques offrent, depuis 2000, des formations parallèles payantes. Ces filières connaissent un très vif succès puisqu'elles représentent environ $40 \%$ des effectifs des universités. Ce mouvement de privatisation de l'enseignement supérieur modifie radicalement le rapport aux savoirs. Les universités s'adaptent en effet aux besoins et aux demandes de l'étudiantclient. Dans ce nouveau contexte, l'éducation tend à devenir un investissement individuel et non plus collectif.

Au total, les années 1980-2000 représentent une rupture majeure dans l'histoire de la scolarisation du Kenya. Au-delà de la crise elle-même qui se traduit par des phénomènes inédits de déscolarisation, la synergie entre l'État et les communautés locales, sur laquelle avait reposé l'essor de la scolarisation depuis l'indépendance, est rompue. L'école risque alors d'aggraver les inégalités sociales avec le développement d'un secteur privé d'éducation attractif. La frustration face à un système scolaire qui ne remplit plus son rôle de régulateur social tend à s'exprimer à travers des formes inédites de violences scolaires (viols collectifs, incendies criminels, etc.) (Charton, 2003).

\section{Le Kenya en marche vers la scolarisation universelle}

L'histoire politique du Kenya connaît un tournant significatif en 2002 avec l'accès au pouvoir d'un président, issu de la coalition d'opposition (National Rainbow Coalition-NaRC), incarnant une véritable volonté de changement et de rupture. Confirmant sa promesse électorale, le nouveau président, Mwai Kibaki, instaure la gratuité de l'éducation primaire dès la rentrée 2003. La décision d'étendre la gratuité 
de l'éducation au niveau secondaire en 2008 s'inscrit dans une même logique électorale. Ces mesures, qui interviennent après quinze années de marasme et de crise, sont accueillies avec un très grand enthousiasme. La concertation et l'aide internationale jouent un rôle décisif dans la mise en œuvre de ces programmes qui s'inscrivent dans la stratégie globale de réduction de la pauvreté. Il s'agit notamment de rendre l'école accessible aux enfants des rues et aux enfants issus des familles les plus pauvres, dont le nombre est estimé à trois millions et demi.

Les écoles ouvrent leurs portes dans une certaine confusion en janvier 2003 avec un million d'élèves supplémentaires et 500000 de plus à la fin du mois de janvier. Le coût direct de l'éducation gratuite est estimé à 62 millions de dollars US, auxquels s'ajoutent 100 millions de dollars pour employer cinquante mille enseignants (il en manquait déjà vingt mille avant 2003). Le gouvernement a débloqué 69,5 millions de dollars US. Pour le reste, les parents continuent à financer la construction et l'entretien des bâtiments scolaires, mais le gouvernement compte surtout sur l'aide de la communauté internationale. La stratégie sectorielle d'éducation (Kenya Education Sector Support Programme [KESSP]), qui doit permettre au Kenya d'atteindre les Objectifs du millénaire et notamment de pérenniser l'éducation universelle, est adoptée en juin 2005 par les bailleurs. Le Kenya est également bénéficiaire de l'initiative Fast Track qui appuie la mise en ouvre de la stratégie sectorielle pour un montant total de 73 millions de dollars US.

L'initiative du gouvernement en faveur de l'éducation primaire universelle s'inscrit dans une dynamique glo- bale qui dépasse les seuls enjeux nationaux. D'après les enquêtes de l'Unesco, le premier bilan est plutôt positif. En 2007, les écoles primaires formelles accueillaient 8,1 millions d'enfants contre 6 millions en 2002, soit un taux net de scolarisation de $91,6 \%$. Le programme d'éducation universelle a permis de ramener vers l'école des jeunes qui avaient dépassé l'âge scolaire ${ }^{5}$. Les taux d'achèvement se sont également améliorés, passant de $58 \%$ à $76,8 \%$ entre 2003 et 2007 (Unesco, 2008). Le ratio moyen enseignant/élève qui était de 1 pour 50 en 2003 est retombé à 1 pour 44 en 2005. La scolarisation universelle a cependant du mal à atteindre les « poches de pauvreté » que constituent les bidonvilles et les zones arides et semi-arides du pays, où plus d'un million d'enfants ne sont pas scolarisés. La stratégie sectorielle prévoit d'aider ces régions à travers un programme d'investissement destiné à quatre mille écoles à hauteur de 100000 à 200000 Ksh (1250 à 2500 dollars US) par an pendant cinq ans.

Un certain nombre de malentendus et de flottements subsistent cependant entre les différents partenaires de l'éducation (État central et décentralisé, parents, communautés et enseignants) sur le contenu exact du programme et les responsabilités de chacun dans sa mise en œuvre. La gratuité n'est pas totale : les communautés continuent de contribuer au développement et à l'entretien des infrastructures scolaires et les parents participent indirectement à l'éducation de leurs enfants (repas, activités, uniformes, fournitures). Ces diverses contributions sont un frein à la généralisation de l'éducation, notamment au niveau secondaire.

5. $44 \%$ des élèves ne seraient pas scolarisés dans la classe correspondant à leur âge (Unesco, 2005). 
Le ministère estime, en effet, que $30 \%$ des abandons scolaires sont directement liés au coût de l'éducation. La décision prise par le gouvernement, en janvier 2008, de prendre en charge les frais de scolarité des élèves du secondaireà hauteur de $10000 \mathrm{Ksh}$ (soit 130 dollars US) devrait permettre d'améliorer les taux de transition entre les deux cycles qui doivent être portée à $70 \%$ (ils étaient de $47 \%$ en 2002). Les frais de pension restent cependant à la charge des familles, cequi représente un coût important car la plupart des lycéens sont scolarisés dans des internats. Pour pallier ces difficultés, le gouvernement s'est engagé à développer les externats ; les élèves les plus nécessiteux bénéficient de bourses d'études confiées aux Constituency Development Funds. Ces mesures en faveur de l'enseignement secondaire constituent une avancée significative en faveur de la 144 scolarisation universelle, et répondent notamment à la proposition formulée en 2004 par 66 représentants d'ONG, d'universités et de syndicats dans un texte intitulé Enhancing transition in education forprimary schoolleavers in Kenya.

Si la «gratuité » de l'enseignement secondaire a été annoncée dès le mois de juillet 2007 par le président et candidat Kibaki, sa mise en œuvre est intervenue au plus fort de la poussée de violence qui a suivi les résultats des élections présidentielles de décembre 2007. Au-delà de ses effets directs sur la scolarisation (destructions d'écoles, déplacements de populations, etc.), la crise a freiné la croissance économique qui avait favorisé les engagements pris en faveur de la scolarisation universelle primaire et secondaire. En 2007, le Kenya avait atteint un taux de croissance de $7 \%$. Or la crise de 2008 a rompu l'équilibre qui reposait précisément sur la confiance accordée à l'équipe dirigeante.
Malgré les incertitudes qui pèsent sur l'avenir politique du pays, le Kenya semble bien engagé sur la voie de l'éducation universelle. Celle-ci reste, en effet, un idéal politique absolu, comme en témoignele calendrier des initiatives prises en ce sens qui ont scandé la vie politique du pays. Mais il s'agit aujourd'hui d'un objectif global, relevant d'un agenda international orchestré par l'État et dont les populations ne sont plus les acteurs principaux. La fragmentation du paysage scolaire amorcée depuis les années 1980 donne un tout autre sens à la scolarisation universelle. L'objectif à atteindre est avant tout quantitatif : $100 \%$. Le principe idéal d'une école universelle comme lieu central de la construction nationale, qui avait soustendu les efforts précoces déployés par le Kenya en faveur de la scolarisation universelle, est relégué au second plan.

\section{Hélène Charton}

\section{RÉFÉRENCES}

ABREU E. (1982): The role of Self help in the development of Education in Kenya: 19001973, Kenya Literature Bureau, Nairobi.

BOGONKO S.N. (1992): A history of modern education in Kenya (1895-1991), Nairobi, Evans brothers, Kenya.

CHARTON H. (2003) : " La débâcle éducative du Kenya ; éléments d'analyse historique » in Cahiers d'études africaines, XLIII, 169-170, 2003, pp. 189-208.

ESHIWANI G. S. (1993) : Education in Kenya since Independence, East African Education Publishers, Nairobi.

KOECH D.K. (2000): Totally integrated quality education and training TIQUET, Report of the Commission of Inquiry into the Education system of Kenya, Nairobi, Government printer.

UNESCO (2005): Implementing free primary education in Kenya, assessment report, Nairobi, March.

UNESCO (2008): Factbook for Unesco national education support strategies, Kenya. 